\title{
Ultrasonography and the American Thyroid Association Ultrasound-Based Risk Stratification Tool: Utility in Pediatric and Adolescent Thyroid Nodules
}

\author{
Ana $\mathrm{Creo}^{\mathrm{a}}$ Fares Alahdab $^{\mathrm{b}}$ Alaa Al Nofal ${ }^{\mathrm{c}}$ Kristen Thomas $^{\mathrm{d}}$ Amy Kolbe $^{\mathrm{d}}$ \\ Siobhan T. Pittock ${ }^{\mathrm{a}}$ \\ ${ }^{a}$ Division of Pediatric Endocrinology and Metabolism, Mayo Clinic, Rochester, MN, USA; ${ }^{b}$ Division of Preventative \\ Medicine, Mayo Clinic, Rochester, MN, USA; ' Division of Pediatric Endocrinology, Sanford Health, Sioux Falls, SD,

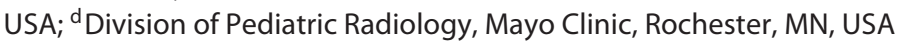

\section{Keywords}

Thyroid nodules · Thyroid cancer · Pediatrics · Children ·

Ultrasonography

\begin{abstract}
Background: Pediatric thyroid nodules are more likely to be malignant compared to those in adults and may have different concerning ultrasound (US) features. Recent adult guidelines stratify malignancy risk by US features. Our aim is to (1) describe and confirm US features that predict pediatric malignancy, and (2) apply the Adult American Thyroid Association (ATA) Risk Stratification Guidelines to a large pediatric cohort. Methods: We identified 112 children with 145 thyroid nodules from 1996 to 2015 . Two blinded pediatric radiologists independently read all US images, described multiple features, and reported their overall impression: benign, indeterminate, or malignant. Each nodule was assigned an ATA risk stratification category. Radiologists' impressions and ATA risk stratification were compared to histology and cytology results. Results: Multiple US features including a solid composition, presence of microcalcifications, irregular margins, increased blood flow, and hypoechogenicity were associated with increased odds of ma-
\end{abstract}

\section{KARGER}

(c) 2018 S. Karger AG, Basel

E-Mail karger@karger.com

www.karger.com/hrp lignancy. ATA risk stratification correlated with the radiologists' overall impression $(p<0.001)$. The sensitivity for detecting malignancy was comparable between both ATA stratification (91\%) and the radiologists' overall impression (90\%). The specificity of the radiologists' malignant overall impression (80\%) was better compared to the ATA high risk stratification (54\%). Conclusions: At our institution, pediatric radiologists' overall impressions had similar sensitivity but better specificity for detecting malignancy than the ATA risk stratification tool by our convention. However, neither US-based methods perfectly discriminated benign from malignant nodules, supporting the continued need for fine needle aspiration for suspicious nodules. Further work is needed to develop an US-based scoring system specific to pediatric patients.

(c) 2018 S. Karger AG, Basel

\section{Introduction}

Thyroid cancer is the most common pediatric endocrine cancer [1]. When a child presents with a thyroid nodule, that nodule is at least $2-4$ times more likely to be malignant compared to that in an adult [2-7]. Recent ma- 
lignancy rate estimates in children with thyroid nodules range from 18 to $26 \%$ (up to $40 \%$ in children with a history of radiation exposure) compared to $5-10 \%$ in adults [2-7]. Furthermore, children often have more advanced cancer at the time of diagnosis compared to adults, with greater lymph node extension, metastases, and chance for recurrence $[1,8,9]$.

In 2015, the American Thyroid Association (ATA) published the Management Guidelines for Children with Thyroid Nodules and Differentiated Thyroid Cancer [10]. These valuable guidelines recommend performing a fine needle aspiration (FNA) in any child with a concerning clinical history (i.e., history of radiation, genetic syndrome, or young age at presentation) or ultrasound (US) finding. Multiple smaller pediatric studies identify consistent US features concerning for malignancy [2]. Suspicious US features are similar between pediatrics and adults [11]. However, we do not have any formalized, USbased risk assessment tool in pediatrics akin to the ATA Adult US Risk Stratification scheme [11]. The adult stratification relies on specific US features to stratify malignancy risk including the presence of hypoechogenicity, irregular margins, calcifications, increased blood flow, suspicious lymph nodes, and larger size.

Recently, Lim-Dunham et al. [12] applied the Adult ATA Risk Stratification to a small cohort of children with good results. Martinez-Rios et al. [13] compared the performance of the ATA Risk Stratification to adult Thyroid Imaging, Reporting, and Data System (TIRADS) scoring in children, validating a similar sensitivity and specificity to adult patients. Likewise, Canfarotta et al. [3] described good diagnostic accuracy of the Adult McGill Criteria (a scoring system based upon clinical, US, and cytology results) in a pilot study of children [14]. Further work is needed to determine if adult US features concerning for malignancy and risk stratification schemes apply equally well to pediatric thyroid nodules. Our goal is to (1) describe and confirm US features that predict pediatric malignancy, and (2) apply the Adult ATA Risk Stratification Guidelines to a large pediatric cohort.

\section{Methods}

\section{Study Design and Setting}

We established a retrospective cohort by identifying pediatric patients ( $\leq 21$ years old) who presented to a single-institution, tertiary care children's center with a thyroid nodule between 1996 and 2015. Data and images were de-identified and abstracted from the medical record. The Mayo Clinic Institutional Review Board approved this study.

\section{Subjects}

We identified 330 patients who presented with nodules during the study period. We elected to include all patients $<21$ years of age, as this was felt to best represent a typical pediatric subspecialty practice. To be included, patients needed to have an initial US performed at Mayo Clinic followed by either (1) histopathology results after thyroidectomy, (2) FNA biopsy cytology results with a follow-up FNA performed at our institution $\geq 1$ year after initial biopsy, (3) US FNA biopsy cytology results with a stable follow-up US performed at our institution $\geq 1$ year after initial biopsy, or (4) stable follow-up US performed at our institution $\geq 1$ year after initial US. We excluded patients with a genetic syndrome known to increase thyroid cancer risk and those who had a history of radiation exposure. While most patients just had 1 nodule, in those with multiple nodules we only included the patient's 2 largest nodules in the analysis in an attempt to reduce any statistical clustering effect. Ultimately, we included 145 thyroid nodules from 112 patients in the cohort.

\section{Ultrasonography}

Diagnostic gray-scale US with color Doppler was obtained using high-frequency linear array transducers. Both cine and still imaging were recorded using longitudinal and transverse views. Cervical lymph nodes were briefly evaluated, and more detail to the lateral neck was given when the nodule was concerning. All images were viewed on the same imaging system and no nodules were excluded from the study because of poor image quality. Using these images, 2 pediatric radiologists with a combined 27 years of experience after pediatric radiology fellowship training independently described multiple features. They were blinded to the previous radiology reads, cytology and/or histology results. Our pediatric radiology group has extensive thyroid expertise with over 225 annual thyroid US exams performed. The overall radiologists' interobserver agreement and variability was good (Kappa 0.7 ). When there was a disagreement, the radiologists conducted a separate session to reach a unified consensus.

The radiologists described specific nodule features based upon the TIRADS descriptions for reporting thyroid nodule features [15]. Given our aim to identify features unique to pediatrics, further divisions were added and recorded as follows:

- Composition: cystic $(<25,25-75,76-90$, or $>90 \%)$; solid $(<25$, $25-75,76-90$, or $>90 \%$ ); spongiform ( $\geq 90 \%$ distinct spongy appearance).

- Margins: regular or irregular ( $\geq 25 \%$ of the margin irregular).

- Calcifications: any calcification of any size, present or absent.

- Echogenicity: hypoechoic, isoechoic, or hyperechoic, mixed (elements of both hypo- and hyperechogenicity) compared to background thyroid tissue or strap muscle if abnormal.

- Taller than wide: present or absent (anteroposterior $>$ transverse dimensions).

- Blood flow: increased centrally, peripherally, or overall (either central or peripheral) based upon dominant Doppler pattern.

- Overall impression: malignant, indeterminate, or benign.

After the radiologists recorded the features, an independent reviewer assigned each nodule a level of suspicion for malignancy based on the 2015 ATA Adult Risk Stratification Guidelines (Table 1). The radiologists' overall impression was based upon the expert overall clinical impression after reviewing the thyroid nodule image on ultrasonography. Radiologists were simply asked to provide their overall impression and were given the de- 
Table 1. ATA ultrasound features and malignancy risk stratification [11]

\begin{tabular}{llll}
\hline $\begin{array}{l}\text { Sonographic } \\
\text { pattern }\end{array}$ & US features & $\begin{array}{l}\text { Estimated } \\
\text { malignancy risk, \% }\end{array}$ & FNA size cutoff \\
\hline High suspicion & $\begin{array}{l}\text { Solid hypoechoic nodule or solid hypoechoic component of a } \\
\text { partially cystic nodule with 1 or more of the following features: } \\
\text { irregular margins (infiltrative, microlobulated) } \\
\text { microcalcifications, taller than wide shape, rim calcifications } \\
\text { with small extrusive soft tissue component, evidence of } \\
\text { extrathyroidal extension (ETE) }\end{array}$ & $>70-90$ & FNA at $\geq 1 \mathrm{~cm}$ \\
\hline $\begin{array}{l}\text { Intermediate } \\
\text { suspicion }\end{array}$ & $\begin{array}{l}\text { Hypoechoic solid nodule with smooth margins without } \\
\text { microcalcifications, ETE, or taller than wide shape }\end{array}$ & $10-20$ & FNA at $\geq 1 \mathrm{~cm}$ \\
\hline Low suspicion & $\begin{array}{l}\text { Isoechoic or hyperechoic solid nodule, or partially cystic nodule } \\
\text { with eccentric solid areas, without microcalcification, irregular } \\
\text { margin or ETE, or taller than wide shape }\end{array}$ & $5-10$ & FNA at $\geq 1.5 \mathrm{~cm}$ \\
\hline $\begin{array}{l}\text { Very low } \\
\text { suspicion }\end{array}$ & $\begin{array}{l}\text { Spongiform or partially cystic nodules without any of the } \\
\text { sonographic features described in low, intermediate, or high } \\
\text { suspicion patterns }\end{array}$ & $<3$ & $\begin{array}{l}\text { Consider FNA at } \geq 2 \mathrm{~cm} ; \\
\text { observation without }\end{array}$ \\
\hline $\begin{array}{l}\text { Benign } \\
\text { Purely cystic nodules (no solid component) }\end{array}$ & FNA is also acceptable \\
\hline
\end{tabular}

scriptive choices of benign, indeterminate, or malignant. The specific elements informing the radiologists' overall impression included the presence or absence of calcifications, the type of margins, as well as the size and composition of the nodules. It was challenging to completely detail and quantify all the intangible factors that went into forming the expert radiologists' overall impression. However, any calcification greatly raised their suspicion for malignancy: in addition, $>25 \%$ and especially $>50 \%$ of the margin being irregular was highly concerning. While the composition influenced their decision-making, they felt that the calcifications and margins had more weight in their decision-making process.

\section{Cytology and Histology}

FNA was performed by institutional radiologists by free-hand technique with US guidance. Cytology results were reported using the Bethesda System for Reporting Thyroid Cytology [16]. This includes (I) nondiagnostic, (II) benign, (III) atypia of undetermined significance, (IV) suspicious for follicular neoplasm, (V) suspicious for malignancy, and (VI) malignant categories. In a child with concerning cytology results who underwent thyroidectomy, histology replaced cytology results. When a child's cytology did not require thyroidectomy, appropriate follow-up with either repeat FNA or repeat US $\geq 1$ year was used to ensure the nodule was accurately classified as benign.

\section{Statistical Analysis}

Statistical analysis was conducted using Microsoft Excel, MedCalc, and JMP. Descriptive statistics included demographic and clinical characteristics by cytology and histology. Diagnostic analyses were conducted yielding sensitivity, specificity, positive and negative predictive values, and diagnostic likelihood ratios. Testspecific analysis was performed using Excel formulas for odds ratio
(OR), CI, and significance from typical contingency tables. $p$ values were based upon 2-tailed testing or Fischer's exact test with statistical significance defined as a $p$ value $<0.05$.

\section{Results}

Our overall malignancy rate was 34\% (Table 2). A total of $44 \%$ of malignant nodules had lymph node metastases at the time of diagnosis.

No single clinical characteristic was associated with malignancy and clinical variables were quite evenly distributed amongst malignant and benign groups. The cohort was predominantly adolescent with a mean age at diagnosis of $15.5 \pm 3.2$ years with few young children $(8 \%$ were $<10$ years old) and some young adults (25\% were 19-21 years old). Most patients in our sample were female (86\%) with sex being equally distributed in those with benign and malignant nodules. On average, $30 \%$ of patients had more than 1 nodule at the time of the first US at our institution. The mode in which the nodule was found, including by patient, provider, or on incidental imaging, was not associated with malignancy. Nodules were first found almost equally by patients and providers (34 and $36 \%$, respectively). A total of $16 \%$ of benign and malignant nodules were found on incidental imaging with 6 of the malignant nodules discovered incidentally. Incidental imaging most commonly included head and neck CT 
Table 2. Characteristics of children with thyroid nodules

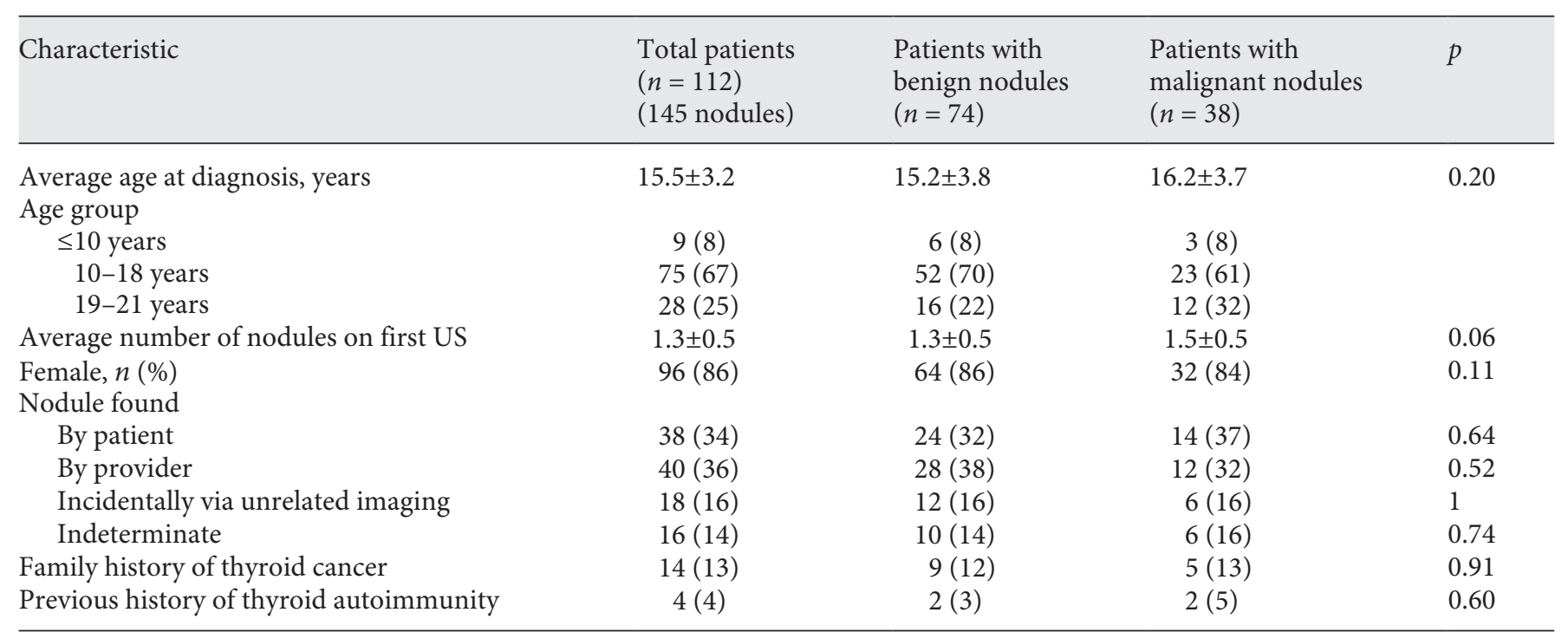

Values are shown as $n(\%)$ or mean \pm SD.

Table 3. Univariate US characteristics and detection of malignancy

\begin{tabular}{|c|c|c|c|c|c|c|c|}
\hline Characteristic & $\begin{array}{l}\text { Benign } \\
(n=95)\end{array}$ & $\begin{array}{l}\text { Malignant } \\
(n=50)\end{array}$ & $\begin{array}{l}\text { Sensitivity, } \\
\%\end{array}$ & $\begin{array}{l}\text { Specificity, } \\
\%\end{array}$ & $\begin{array}{l}\text { Odds } \\
\text { ratio }\end{array}$ & $95 \% \mathrm{CI}$ & $p$ \\
\hline \multicolumn{8}{|l|}{ Composition } \\
\hline$>75 \%$ solid & $61(64)$ & $42(84)$ & 84 & 36 & 2.9 & $1.2-6.9$ & $0.01^{*}$ \\
\hline Spongiform & $11(12)$ & 0 & & & 0.07 & $0.0042-1.2$ & 0.07 \\
\hline Size $>1 \mathrm{~cm}$ & $70(74)$ & $38(76)$ & & & 1.1 & $0.5-2.5$ & 0.76 \\
\hline Irregular margins present & $44(46)$ & $35(70)$ & 70 & 54 & 2.7 & $1.3-5.6$ & 0.0073 \\
\hline Suspicious lymph node(s) present & $3(3)$ & $20(40)$ & 40 & 97 & 20.4 & $5.6-73.6$ & $<0.0001^{*}$ \\
\hline Halo present & $11(12)$ & $7(14)$ & & & 0.8 & $0.3-2.2$ & 0.67 \\
\hline Taller than wide & $6(6)$ & $7(14)$ & 14 & 94 & 2.4 & $0.7-7.6$ & 0.13 \\
\hline \multicolumn{8}{|l|}{ Blood flow } \\
\hline Increased central & $49(52)$ & $34(68)$ & & & 2.0 & $1.0-4.1$ & 0.06 \\
\hline Hyperechogenic & $74(78)$ & $36(72)$ & & & 0.7 & $0.3-1.6$ & 0.43 \\
\hline Mixed & $28(29)$ & $23(46)$ & 46 & 71 & 2.0 & $1.0-4.1$ & $0.049^{*}$ \\
\hline
\end{tabular}

Values are shown as $n(\%)$, unless otherwise indicated.

scans and occasional neck US for nonthyroid related concerns. Although patients with a genetic syndrome associated with thyroid cancer were excluded, 14/112 patients (13\%) had a family history of thyroid cancer. Those who did have a family history were no more likely to have malignant nodules compared to those without a family history of thyroid cancer. Lastly, $4 \%$ of patients had a previously documented history of thyroid autoimmunity de- 


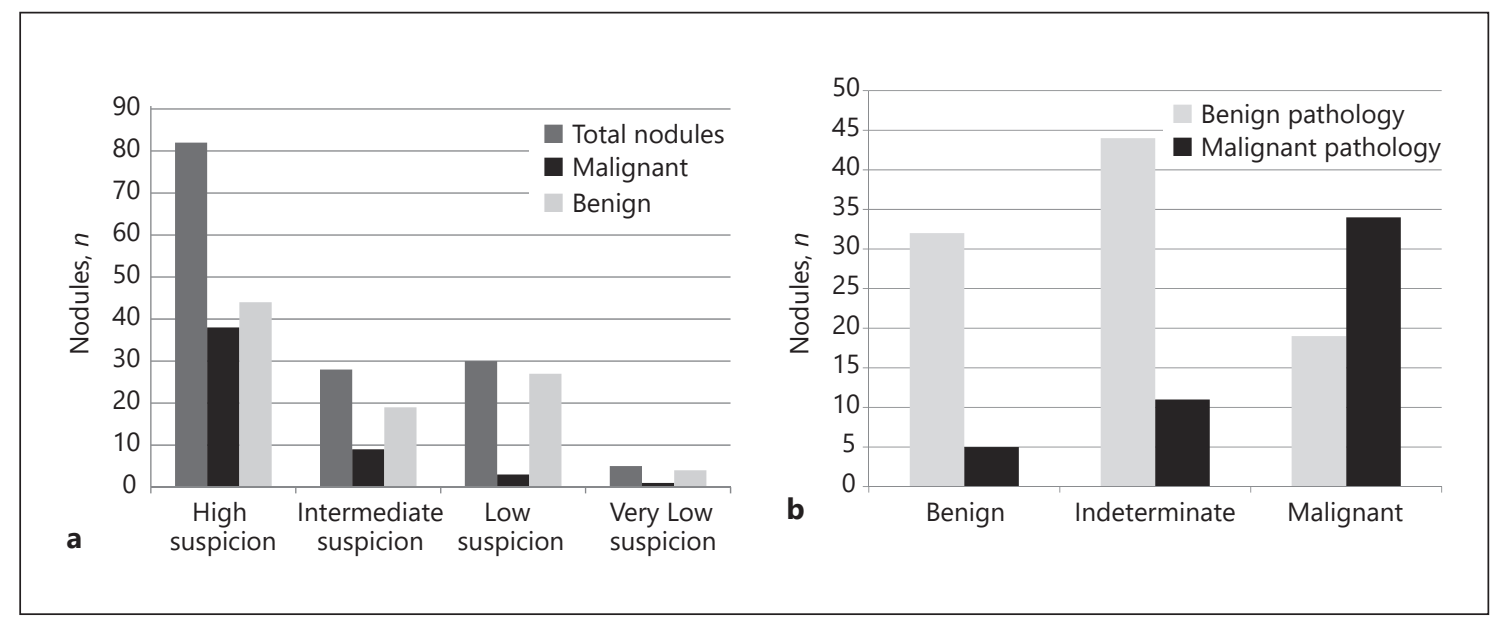

Fig. 1. Nodule distribution amongst ATA risk level (a) and radiologists' overall impression (b).

fined by previously abnormal thyroid peroxidase or thyroid-stimulating antibodies; its presence was not associated with malignancy.

When examining specific US features, the presence of calcifications, suspicious lymph nodes, a solid component, and hypoechogenicity were all important for predicting malignancy (Table 3). The following features predicting malignancy included: suspicious lymph nodes (OR 20.4, 95\% CI 5.6-73.6; $p<0.0001$ ), presence of calcifications (OR 6.9, 95\% CI 3.2-14.9; $p<0.0001$ ), irregular margins (OR 2.7, 95\% CI 1.3-5.6; $p=0.0073$ ), hypoechogenicity (OR 2.8, 95\% CI 1.3-5.9; $p=0.0073$ ), $>75 \%$ solid (OR 2.9, 95\% CI 1.2-6.9; $p=0.01$ ), and overall increased nodular blood flow (OR 2.9, 95\% CI 1.2-7.0; $p=0.01$ ). Nodules taller than wide were uncommon (13/145 nodules, 9\%), but when present had an OR for malignancy of 2.4 but this was not statistically significant (95\% CI $0.7-$ $7.6, p=0.13$ ), likely due to the small sample size.

Features not significantly predictive of malignant or benign disease included spongiform morphology (OR $0.07,95 \%$ CI $0.0042-1.2 ; p=0.07)$, hyperechogenicity (OR $0.7,95 \%$ CI $0.3-1.6 ; p=0.43)$, presence of a halo (OR $0.8,95 \%$ CI $0.3-2.2 ; p=0.67)$, and size $>1 \mathrm{~cm}$ (OR 1.1, $95 \%$ CI $0.5-2.5 ; p=0.76$ ).

Most malignant nodules were categorized in the ATA high or intermediate suspicion groups (Fig. 1a). In our cohort, 81/145 (56\%) nodules were categorized as high suspicion, of which $37 / 81$ (46\%) were malignant. A total of 28/145 (19\%) nodules were categorized as intermediate suspicion, of which $9 / 28$ (32\%) were malignant. However, $4 / 50(8 \%)$ malignant nodules were categorized as low or

Risk Stratification in Pediatric Thyroid Nodules

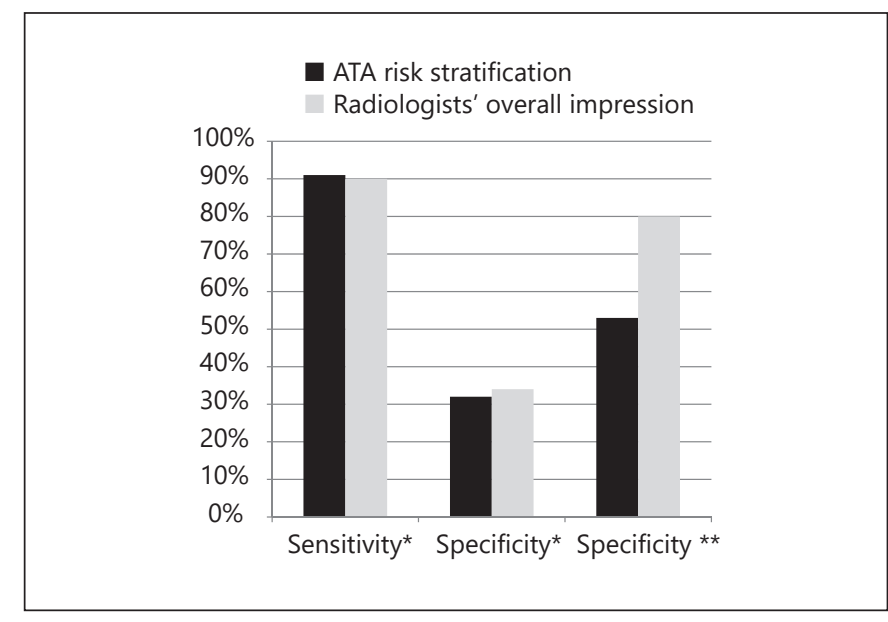

Fig. 2. Diagnostic accuracy of ATA risk stratification versus radiologists' overall impression. * ATA high and intermediate suspicion versus radiologists' malignant and indeterminate overall impression. ** ATA high suspicion versus radiologists' malignant overall impression.

very low suspicion. The radiologists called 53/145 (37\%) nodules malignant, of which 34/53 (64\%) were malignant (Fig. 1b). A total of 55/145 (38\%) nodules were called indeterminate, of which 11/55 (20\%) were malignant. Five out of $50(10 \%)$ malignant nodules were misclassified as benign. In summary, while the radiologists' impression was more accurate than risk stratification, there were still a substantial number of nodules assigned an incorrect overall impression. 
Next, we compared the diagnostic accuracy of the ATA risk stratification to the radiologists' overall impression (Fig. 2). In clinical practice, an FNA would typically be performed for a high or intermediate ATA risk stratification or a radiologist's malignant or indeterminate overall impression. Therefore, the sensitivity of the ATA high and intermediate suspicion groups was compared to the sensitivity of the radiologists' malignant and indeterminate impression groups to best answer the practical clinical question of how many malignant nodules would be missed if an FNA was not performed. Using this convention, the sensitivity for detecting malignancy was equal between both ATA criteria and radiologists' interpretation (91 and 90\%, respectively). When we compared the specificity of the ATA high and intermediate categories compared to the radiologists' malignant and indeterminate overall impressions, the specificity was also comparable (32\% ATA and 34\% radiologists). However, when we compared only the ATA high suspicion and the radiologists' malignant impression, the specificity of the radiologists was higher than the ATA risk stratification ( $80 \mathrm{vs.}$ $53 \%$, respectively). When a subset analysis was performed on only patients $<18$ years old, the results were similar: the overall malignancy rate was $31 \%$ (compared to $34 \%$ in the $<21$-year-old group). The ATA risk stratification sensitivity and specificity was 87 and $54 \%$, respectively, compared to 86 and $81 \%$ for the radiologists' overall impression.

\section{Discussion}

In this study, $34 \%$ of children presenting with thyroid nodules had thyroid cancer. Other groups have reported a slightly lower malignancy rate for pediatric thyroid nodules ranging from 18 to $26 \%[2,4,6]$. Our estimate likely inflates the malignancy rate due to both a referral bias and the intentional study design requiring at least 1 year of institutional follow-up, likely eliminating more patients with benign nodules who chose to have followup care closer to home. No single clinical characteristic, including age, sex, mode of presentation, or family history of thyroid malignancy, was significantly different between patients with benign and malignant nodules. Our cohort was predominantly female, similar to previous studies $[6,17,18]$. Interestingly, we did not find any association with the mode of nodule discovery and malignancy. Previous work suggested thyroid nodules found on incidental imaging may be less likely to be malignant, but our results do not support this conclusion [19]. Inci- dental nodules were discovered most frequently during head CT scans and neck US imaging. Our practice has been to consider FNA for any nodules $\geq 5 \mathrm{~mm}$ with concerning features regardless of the reason for imaging. Our study did not capture the patients with benign-appearing nodules who had follow-up locally; the proportion of these nodules which were found incidentally is unknown. Nevertheless, of the 12 nodules in this study discovered on incidental imaging, 50\% were malignant supporting that pediatric thyroid nodules, even if found incidentally, require further investigation. In our study, few patients (4\%) had thyroid autoimmunity, defined as having positive thyroid antibodies. Given this study was a retrospective review, it is possible that more patients in this cohort may have had measurable thyroid antibodies, but did not have antibody tests performed. Thyroid antibodies are not ordered routinely in euthyroid patients presenting with thyroid nodules or cancer. Our study supports previous work that pediatric cancer often is more advanced at the time of diagnosis: $46 \%$ of children had evidence of lymph node involvement at diagnosis [19].

We identified US characteristics associated with increased odds of thyroid malignancy, these included: suspicious lymph nodes, calcifications, irregular margins, hypoechogenicity, a significant solid component, and increased blood flow. Nodules that were spongiform, had a halo, or were hyperechogenic decreased the odds of malignancy, but did not reach statistical significance potentially due to the fact that these features were infrequently present. As previously described, we confirmed that the presence of suspicious lymph node involvement highly predicted malignancy $[4,8,19-22]$. The presence of microcalcifications has been one of the US features most consistently associated with malignancy across many studies, and our study maintains this strong association [2].

Our results also support previous work suggesting that irregular margins reflect increased odds of malignancy [23]. As the definition of an irregular margin has not been clearly defined in previous studies, we found $\geq 25 \%$ of the margin irregular predictive for malignancy and suggest this be used as a formal definition. Our study reported on degrees of solidity and found that $>75 \%$ solid nodules had an OR of 2.9 (95\% CI 1.2-6.9, $p=0.01$ ). We propose using a definition of $>75 \%$ solid in future work.

Echogenicity has been weakly linked to malignancy risk $[19,20,24]$. We found that predominantly hypoechoic nodules without any hyperechoic component strongly predicted malignancy risk (OR 2.8, 95\% CI 1.3-5.9; $p=$ $0.0073)$. Overall increased vascularity predicted malig-
98

Horm Res Paediatr 2018;90:93-101 DOI: $10.1159 / 000490468$
Creo/Alahdab/Al Nofal/Thomas/Kolbe/ Pittock 
nancy risk (OR 2.9, 95\% CI 1.2-7.0; $p=0.01$ ) as in previous studies [23]. Our work suggests that size $>1$ or $<1 \mathrm{~cm}$ did not predict increased odds of malignancy. There have been conflicting prior studies around this question, but our finding supports the current ATA Pediatric Guidelines which suggest performing FNA of nodules with concerning features even if they are $<1 \mathrm{~cm}$ in diameter $[4,17$, $19,20,25-27]$. Overall, we found the summation of US features concerning for pediatric malignancy was very similar to adult data $[28,29]$.

The overall sensitivity of both the ATA Risk Stratification tool and the radiologists' overall impression was good (91 and 90\%, respectively). Recent studies cite slightly lower US diagnostic accuracy with a lower sensitivity but equal specificity $[30,31]$. The radiologists had a better ability to confidently predict a true malignancy compared to the ATA high risk category ( 80 vs. $53 \%$ ). However, specificity was reduced and similar in both groups when ATA high and intermediate risk or radiologists' malignant and indeterminate categories were combined. Importantly, malignant nodules were misclassified by both groups: 4/50 malignant nodules were stratified as low risk by ATA criteria, and 5/50 were read as benign by the radiologists. This rate of misclassification is likely inflated due to our study design when many patients with benign appearing nodules did not have follow-up at our institution.

However, the overall rate of misclassified thyroid nodules (both falsely benign and malignant nodules) using USbased methods remains small but substantial. Our data, with nearly $10 \%$ of malignant nodules categorized as benign or low risk, highlight the importance of ensuring longitudinal follow-up for thyroid nodules in children even if initially thought to be benign. It is possible to consider avoiding FNA in very low suspicion nodules if providers can ensure appropriate and timely surveillance. Also, given the small but still significant number of nodules misclassified as malignant, we recommend proceeding with FNA prior to surgery in all cases to ensure accurate surgical planning and avoiding overly aggressive surgery.

Another scoring scheme, the McGill Criteria, considers clinical, US, and cytology results to estimate a nodule's malignancy risk [3]. The diagnostic performance of a modified Adult McGill Thyroid Nodule Score in 46 children (10 children with malignant nodules) had a $100 \%$ sensitivity and $94 \%$ specificity for scores $\geq 10$ and $80 \%$ sensitivity and $100 \%$ specificity for scores $\geq 11$ (maximum possible score is 31$)[3,14]$. Specific US variables and clinical factors significantly associated with malignant cytology included presence of a TSH $>1.4 \mathrm{mIU} / \mathrm{L}$,

Risk Stratification in Pediatric Thyroid Nodules hypoechogenicity, microcalcifications, and lymphadenopathy. While the diagnostic accuracy of the McGill Criteria is excellent, a significant limitation is that the score requires an FNA, which independently predicts thyroid cancer in children [32-34].

Developing a scoring system entirely based on noninvasive measures would be ideal for the pediatric population. An US-based stratification system may have the most clinical utility in pediatrics as obtaining cytology results is costly and frequently requires sedation with potentially higher risk and psychological stress for children compared to adults. Other work supports the potential of US-based ATA risk stratification. Lim-Dunham et al. [12] described an excellent diagnostic performance of ATA Adult Risk Stratification in a small cohort of 33 children (12 of whom had malignant nodules). The authors concluded that US surveillance may be appropriate for pediatric nodules stratified to less than high suspicion groups. US is also appealing with low intraobserver variability in performance [35]. It may be also possible to integrate an US stratification scheme with an adapted version of the TIRADS system as the TIRADS system has been well correlated with FNA results in adults [36]. In addition to US, adding strain elastography may be a helpful and noninvasive way to further categorize adult nodules, this technology needs to be evaluated in children $[37,38]$. Overall, a pediatric US-based stratification system holds promise as an adjunctive tool in the evaluation of pediatric patients with thyroid nodules.

Our results should be interpreted in the context of several limitations. First, we intentionally excluded patients who presented with thyroid nodules and did not have longitudinal follow-up at our institution to increase diagnostic validity. By doing so, we excluded many children with benign nodules who continued follow-up at home institutions after an initial reassuring evaluation. Also, nodules were analyzed apart from patients which could inflate results due to a clustering effect in patients with more than 1 malignant nodule. However, the majority of patients only had 1 thyroid nodule, so we feel this effect, if present, is likely minimal. Lastly, we had the benefit of experienced pediatric radiologists whose diagnostic accuracy may not generalize universally across all radiologists. We also recognize the limited ability to define what fully constitutes the radiologists' overall impression and describe the entire breadth of the radiologists' diagnostic reasoning. It is possible that our radiologists' ability to form highly accurate overall impressions is unique, but we think it is highly likely that with training and experience, it could be reproduced.

Horm Res Paediatr 2018;90:93-101 DOI: $10.1159 / 000490468$ 


\section{Conclusion}

We found a high rate of thyroid cancer amongst pediatric patients presenting with thyroid nodules and, furthermore, many patients had advanced cancer at the time of diagnosis. We did not identify a single clinical feature associated with malignancy. Patients' nodules were discovered by a variety of means, and half of nodules discovered incidentally were malignant. Multiple specific US features including solid composition $(>75 \%)$, presence of microcalcifications, irregular margins ( $\geq 25 \%)$, increased blood flow, and hypoechogenicity were strongly associated with increased odds of malignancy. Applying the Adult ATA US Risk Stratification Scheme to pediatric nodules performed well with an equal sensitivity to experienced radiologists. In our study, radiologists could detect malignancy with increased specificity compared to the ATA Risk Stratification Scheme. Our results demon- strate promising use of US to predict malignancy, potentially reducing unnecessary FNA procedures in very lowrisk nodules where follow-up can be ensured. However, both US-based methods were imperfect in detecting malignancy, supporting the continued need for FNA to accurately evaluate most thyroid nodules. Considering the complexity of performing pediatric FNA, developing a formalized US-based system to better predict malignancy and define those nodules requiring FNA would be ideal for this population. Further work is needed to refine an US-based scoring system specific to pediatric patients.

\section{Disclosure Statement}

No authors have any commercial associations which may create a conflict of interest in regard to this paper.

\section{References}

1 Siegel DA, King J, Tai E, Buchanan N, Ajani UA, Li J. Cancer incidence rates and trends among children and adolescents in the United States, 2001-2009. Pediatrics. 2014 Oct; 134(4):e945-55.

2 Al Nofal A, Gionfriddo MR, Javed A, Haydour Q, Brito JP, Prokop LJ, et al. Accuracy of thyroid nodule sonography for the detection of thyroid cancer in children: systematic review and meta-analysis. Clin Endocrinol (Oxf). 2016 Mar;84(3):423-30.

3 Canfarotta M, Moote D, Finck C, Riba-Wolman R, Thaker S, Lerer TJ, et al. McGill Thyroid Nodule Score in Differentiating Benign and Malignant Pediatric Thyroid Nodules: A Pilot Study. Otolaryngol Head Neck Surg. 2017 Oct;157(4):589-95.

4 Gupta A, Ly S, Castroneves LA, Frates MC, Benson CB, Feldman HA, et al. A standardized assessment of thyroid nodules in children confirms higher cancer prevalence than in adults. J Clin Endocrinol Metab. 2013 Aug; 98(8):3238-45.

5 Hung W. Solitary thyroid nodules in 93 children and adolescents. a 35-years experience. Horm Res. 1999;52(1):15-8.

6 Niedziela M. Pathogenesis, diagnosis and management of thyroid nodules in children. Endocr Relat Cancer. 2006 Jun;13(2):427-53.

7 Raab SS, Silverman JF, Elsheikh TM, Thomas PA, Wakely PE. Pediatric thyroid nodules: disease demographics and clinical management as determined by fine needle aspiration biopsy. Pediatrics. 1995 Jan;95(1):46-9.
8 Corrias A, Einaudi S, Chiorboli E, Weber G, Crinò $\mathrm{A}$, Andreo $\mathrm{M}$, et al. Accuracy of fine needle aspiration biopsy of thyroid nodules in detecting malignancy in childhood: comparison with conventional clinical, laboratory, and imaging approaches. J Clin Endocrinol Metab. 2001 Oct;86(10):4644-8.

9 Welch Dinauer CA, Tuttle RM, Robie DK, McClellan DR, Svec RL, Adair C, et al. Clinical features associated with metastasis and recurrence of differentiated thyroid cancer in children, adolescents and young adults. Clin Endocrinol (Oxf). 1998 Nov;49(5): 619-28.

10 Francis GL, Waguespack SG, Bauer AJ, Angelos P, Benvenga S, Cerutti JM, et al. Management Guidelines for Children with Thyroid Nodules and Differentiated Thyroid Cancer. Thyroid. 2015;25:716-759.

11 Haugen BR, Alexander EK, Bible KC, Doherty GM, Mandel SJ, Nikiforov YE, et al. 2015 American Thyroid Association Management Guidelines for Adult Patients with Thyroid Nodules and Differentiated Thyroid Cancer: The American Thyroid Association Guidelines Task Force on Thyroid Nodules and Differentiated Thyroid Cancer. Thyroid. 2016 Jan;26(1):1-133.

12 Lim-Dunham JE, Erdem Toslak I, Alsabban K, Aziz A, Martin B, Okur G, et al. Ultrasound risk stratification for malignancy using the 2015 American Thyroid Association Management Guidelines for Children with Thyroid Nodules and Differentiated Thyroid Cancer. Pediatr Radiol. 2017 Apr;47(4):42936.
13 Martinez-Rios C, Daneman A, Bajno L, van der Kaay DC, Moineddin R, Wasserman JD. Utility of adult-based ultrasound malignancy risk stratifications in pediatric thyroid nodules. Pediatr Radiol. 2018 Jan;48(1):74-84.

14 Sands NB, Karls S, Amir A, Tamilia M, Gologan O, Rochon L, et al. McGill Thyroid Nodule Score (MTNS): "rating the risk," a novel predictive scheme for cancer risk determination. J Otolaryngol Head Neck Surg. 2011 Feb; 40 Suppl 1:S1-13.

15 Grant EG, Tessler FN, Hoang JK, Langer JE, Beland MD, Berland LL, et al. Thyroid Ultrasound Reporting Lexicon: White Paper of the ACR Thyroid Imaging, Reporting and Data System (TIRADS) Committee. J Am Coll Radiol. 2015 Dec;12(12 Pt A):1272-9.

16 Cibas ES, Ali SZ. The Bethesda System for Reporting Thyroid Cytopathology. Thyroid. 2009 Nov; 19(11):1159-65.

17 Drozd VM, Astachova LN, Polyanskaya ON, Schomerus HG, Kaser T, Koritko NL, et al. Characteristics of thyroid ultrasound pictures in children with nodular thyroid changes effected by radionuclides. Bildgebung. 1995 Dec;62(4):236-41.

18 Lugo-Vicente H, Ortíz VN. Pediatric thyroid nodules: insights in management. Bol Asoc Med P R. 1998 Apr-Jun;90(4-6):74-8.

19 Buryk MA, Simons JP, Picarsic J, Monaco SE, Ozolek JA, Joyce J, et al. Can malignant thyroid nodules be distinguished from benign thyroid nodules in children and adolescents by clinical characteristics? A review of 89 pediatric patients with thyroid nodules. Thyroid. 2015 Apr;25(4):392-400. 
20 Corrias A, Mussa A, Baronio F, Arrigo T, Salerno M, Segni M, et al.; Study Group for Thyroid Diseases of Italian Society for Pediatric Endocrinology and Diabetology (SIEDP/ ISPED). Diagnostic features of thyroid nodules in pediatrics. Arch Pediatr Adolesc Med. 2010 Aug;164(8):714-9.

21 Goldfarb M, Gondek SS, Sanchez Y, Lew JI. Clinic-based ultrasound can predict malignancy in pediatric thyroid nodules. Thyroid. 2012 Aug;22(8):827-31.

22 Karaguzel G, Tanyel FC, Buyukpamukcu N, Hicsonmez A. Is there any predictive characteristic for malignancy in thyroid enlargements during childhood? Eur J Pediatr Surg. 1996 Apr;6(2):70-4.

23 Lyshchik A, Drozd V, Demidchik Y, Reiners C. Diagnosis of thyroid cancer in children: value of gray-scale and power doppler US. Radiology. 2005 May;235(2):604-13.

24 Solymosi T, Lukacs Toth G, Budai L, Gal I. The Clinical and Pathological Presentation of Thyroid Nodules in Children and the Comparison with Adult Population: Experience of a Single Institution. Int J Endocrinol. 2016; 2016:1256189.

25 Khozeimeh N, Gingalewski C. Thyroid Nodules in Children: A Single Institution's Experience. J Oncol. 2011;2011:974125.

26 Roy R, Kouniavsky G, Schneider E, Allendorf JD, Chabot JA, Logerfo P, et al. Predictive factors of malignancy in pediatric thyroid nodules. Surgery. 2011 Dec;150(6):1228-33.
27 Koltin D, O’Gorman CS, Murphy A, Ngan B, Daneman A, Navarro OM, et al. Pediatric thyroid nodules: ultrasonographic characteristics and inter-observer variability in prediction of malignancy. J Pediatr Endocrinol Metab. 2016 Jul;29(7):789-94.

28 Brito JP, Gionfriddo MR, Al Nofal A, Boehmer KR, Leppin AL, Reading C, et al. The accuracy of thyroid nodule ultrasound to predict thyroid cancer: systematic review and metaanalysis. J Clin Endocrinol Metab. 2014 Apr; 99(4):1253-63.

29 Remonti LR, Kramer CK, Leitao CB, Pinto LC, Gross JL. Thyroid ultrasound features and risk of carcinoma: a systematic review and meta-analysis of observational studies. Thyroid. 2015 May;25(5):538-50.

30 Mirshemirani A, Roshanzamir F, Tabari AK, Ghorobi J, Salehpoor S, Gorji FA. Thyroid nodules in childhood: a single institute experience. Iran J Pediatr. 2010 Mar;20(1):91-6.

31 Saavedra J, Deladoëy J, Saint-Vil D, Boivin Y, Alos N, Deal C, et al. Is ultrasonography useful in predicting thyroid cancer in children with thyroid nodules and apparently benign cytopathologic features? Horm Res Paediatr. 2011;75(4):269-75.

32 Cole $\mathrm{CD}, \mathrm{Wu} \mathrm{HH}$. Fine-needle aspiration in pediatric patients 12 years of age and younger: a 20 -year retrospective study from a single tertiary medical center. Diagn Cytopathol. 2014 Jul;42(7):600-5.
33 Pantola C, Kala S, Khan L, Pantola S, Singh M, Verma S. Cytological diagnosis of pediatric thyroid nodule in perspective of the Bethesda System for Reporting Thyroid Cytopathology. J Cytol. 2016 Oct-Dec;33(4):220-3.

34 Shirian S, Daneshbod Y, Haghpanah S, Khademi B, Noorbakhsh F, Ghaemi A, et al. Spectrum of pediatric tumors diagnosed by fineneedle aspiration cytology. Medicine (Baltimore). 2017 Feb;96(6):e5480.

35 Park SJ, Park SH, Choi YJ, Kim DW, Son EJ, Lee HS, et al. Interobserver variability and diagnostic performance in US assessment of thyroid nodule according to size. Ultraschall Med. 2012 Dec;33(7):E186-90.

36 Vargas-Uricoechea H, Meza-Cabrera I, Herrera-Chaparro J. Concordance between the TIRADS ultrasound criteria and the BETHESDA cytology criteria on the nontoxic thyroid nodule. Thyroid Res. 2017 Feb;10(1):1.

37 Erkan M, Canberk S, Kilicoglu GZ, Onenerk M, Uludokumaci A, Gunes P, et al. Avoidance of unnecessary fine-needle aspiration with the use of the Thyroid Imaging Reporting Data System classification and strain elastography based on The Bethesda System for Reporting Thyroid Cytopathology. Mol Clin Oncol. 2016 Nov;5(5):625-30.

38 Nell S, Kist JW, Debray TP, de Keizer B, van Oostenbrugge TJ, Borel Rinkes IH, et al. Qualitative elastography can replace thyroid nodule fine-needle aspiration in patients with soft thyroid nodules. A systematic review and meta-analysis. Eur J Radiol. 2015 Apr;84(4): $652-61$. 